\title{
Datengetriebene Diagnose von Regelarmaturen zur Steigerung der Anlagenverfügbarkeit
}

\section{Data-driven Diagnosis of Control Fittings For Increasing Plant Availability}

\author{
Emanuel Trunzer, Birgit Vogel-Heuser, \\ Lehrstuhl für Automatisierung und Informationssysteme, TU München; \\ Christian Vermum, Evonik Industries AG, Marl
}

\section{Kurzfassung}

Trotz hoher Anlagenverfügbarkeiten in der Prozessindustrie sorgen ungeplante Anlagenstillstände für Produktionsausfälle. Beispiel hierfür ist der ungeplante Austausch verschlissener Regelventile, welche ihren sicherheits- oder regeltechnischen Anforderungen nicht mehr nachkommen. Durch die große Zahl an Signalen in chemischen Anlagen bieten die Anwendung von Industrie 4.0-Prinzipien und datengetriebenen Analysen einen Ausweg. Mittels Datenanalyse können Defekte frühzeitig erkannt und präventive Maßnahmen ergriffen werden. Für die industrielle Anwendung müssen jedoch einige Herausforderungen überwunden werden. Dieser Beitrag präsentiert diese Anforderungen für den Anwendungsfall der Diagnose von Regelarmaturen. Derzeitige Hindernisse, wie die Heterogenität und Geschlossenheit der IT-Infrastruktur, die Komplexität zugrundeliegender physikalischer Phänomene und Probleme bei der Datenanalyse, werden thematisiert und Lösungsansätze gezeigt. Im Fokus steht die Nutzbarmachung von Datenanalysetechniken für bestehende Anlagen. Weiterhin wird der Nutzen von geteilten Wissensdatenbanken und Ergebnisse zur Verdeutlichung der Potentiale datengetriebener Analysen gezeigt.

\begin{abstract}
Unplanned downtimes cause production losses in chemical process plants despite an already high degree of availability. One example is the unplanned replacement of control valves that cannot fulfill their safety and control requirements any more. Due to the large number of signals from process plants, the application of Industrie 4.0 principles and datadriven analysis provide a possible solution. With data analysis, defects can be identified early and preventive measures be taken. For industrial application a number of challenges has to be solved. This contribution presents the requirements for the use-case of diagnosis of control fittings. Obstacles, like the heterogeneity and closeness of the IT infrastructure, the com-
\end{abstract}


plexity of physical phenomena and problems during data analysis are discussed and solutions are provided. The application of data analysis techniques in existing production plants is in focus. Furthermore the benefit of shared knowledge pools and results for demonstration of the potential of data-driven analysis are shown.

\section{Industrie 4.0-Prinzipien und Datenanalyse zur Erhöhung der Anlagenverfügbarkeit}

Die Verfügbarkeit prozesstechnischer Anlagen spielt für deren Wirtschaftlichkeit eine entscheidende Rolle. Durch geplante Wartungszyklen und präventive Wartung wird versucht Produktionsausfälle durch ungeplante Anlagenstillstände soweit möglich zu vermeiden. Dies führt zum einen zu einer Überwartung eines Großteils der technischen Einrichtungen. Andererseits sind ungeplante Stillstände aufgrund Versagen bestimmter Einrichtungen weiterhin möglich, da der wirkliche Zustand der Einrichtungen derzeit kaum erfasst wird.

Durch Überwachung der Prozessdaten mittels datengetriebener Verfahren können die Zustände der Einrichtungen beschrieben werden und zielgerichtete, zustandsbasierte Wartungen initiiert werden. Die Anwendung von Big Data-Methoden und Prinzipien der Industrie 4.0 in der Prozesstechnik haben somit das Potential, die Anlagenverfügbarkeit zu erhöhen und Produktionsausfälle zu vermeiden [1]. Jedoch stehen der breiten Anwendung dieser Prinzipien im industriellen Umfeld einige Hindernisse im Weg, welche zunächst überwunden werden müssen.

Dieser Beitrag diskutiert eine Auswahl relevanter Herausforderungen am Beispiel der datengetriebenen Diagnose von Regelarmaturen in der Prozesstechnik und zeigt die innerhalb des Projekts SIDAP entwickelten Lösungswege auf. SIDAP (Skalierbares Integrationskonzept zur Datenaggregation, -analyse, -aufbereitung von großen Datenmengen in der Prozessindustrie) [2] erforscht datengetriebene Diagnosemethoden zur Vorhersage von Geräteausfällen. Hierzu werden relevante Datenquellen über verschiedene Unternehmen und Standorte hinweg benutzt, um den Zustand der technischen Einrichtungen abzuschätzen und vorherzusagen. Diese Daten umfassen neben den aufgezeichneten Sensorwerten beispielsweise Auslegungsdaten und Wartungsdokumentationen.

Ein Anwendungsfall innerhalb von SIDAP ist die Ventildiagnose (vergleiche Bild 1) um Verschleißerscheinungen und Anbackungen am Ventilkegel/-sitz und Packung rechtzeitig zu erkennen. Da Regelarmaturen kritische Aufgaben der Prozessführung übernehmen, führt ein Ausfall dieser Armaturen oftmals zur Abschaltung der Anlage und dem Austausch der Armatur. Die vorzeitige Erkennung und Vorhersage einer möglichen Schädigung ermöglicht die zustandsbasierte Wartung und eine Anpassung der zielgerichteten Wartungszyklen. 


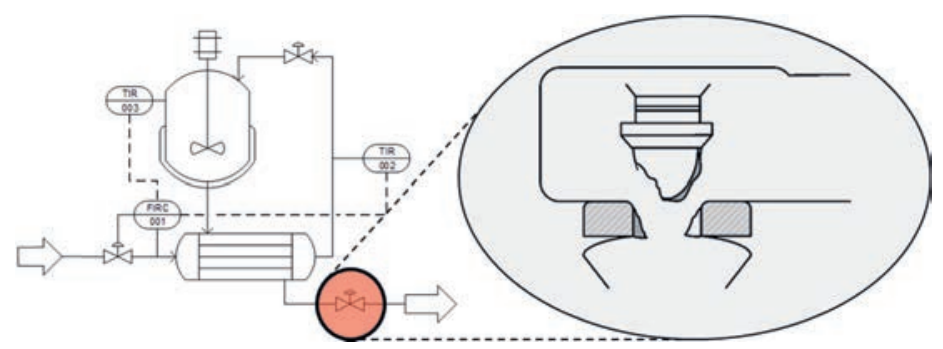

Bild 1: Anwendungsfall Ventildiagnose mit möglichen Fehlerszenarien (Verschlissener Kegel/Sitz (links) und Anbackungen (rechts)

\section{Herausforderungen und Lösungsansätze bei der Umsetzung von datengetriebenen Diagnosen von Regelarmaturen}

Die Anwendung von Industrie 4.0-Prinzipien und von Datenanalyse innerhalb der Prozesstechnik stößt derzeit noch auf mehrere Herausforderungen aus verschiedenen Bereichen. Die konkreten Herausforderungen für ein Unternehmen unterscheiden sich jedoch abhängig von der spezifischen Ist-lage, intern bereits umgesetzten Ansätzen und dem angestrebten Grad der Umsetzung. In diesem Abschnitt werden einige der innerhalb von SIDAP identifizierten Hindernisse und mögliche Lösungswege genannt.

\section{Heterogenität der Datenhaltung}

Verschiedenste Systeme, Werkzeuge und Vertragsparteien begleiten die Auslegungs- und Betriebsphase einer prozesstechnischen Anlage (vergleiche Bild 2). So liegen Betriebsdaten (Sensor- und Aktorsignale) oft in Plant Information Management Systemen (PIMS) beim Betreiber gespeichert vor. Andererseits führt ein Dienstleister im Auftrag des Betreibers verschiedene Wartungsaktionen an Ventilen durch, welche intern dokumentiert werden. Diese Dokumentationen sind derzeit noch oft papierbasiert und werden nicht an den Betreiber der Anlage übermittelt. Des Weiteren werden Daten, welche im Auslegungsprozess der Anlage anfallen nicht aus den CAE-Werkzeugen (Computer Aided Engineering) mit in die Betriebsphase übernommen. Am Übergang zwischen Auslegung und Betrieb findet derzeit oftmals noch ein Bruch in der Datenhaltung statt. Neben den unterschiedlichen Orten der Datenhaltung (verschiedene Systeme) spielten hier auch Unterschiede innerhalb der Struktur der Daten eine Rolle. Während Betriebsdaten meist in relationalen Datenbanken gespeichert werden, liegen die Auslegungsdaten oft nur unstrukturiert in Form von Datenblättern vor. Für die umfassende Analyse von Assets innerhalb einer Anlage ist es jedoch notwendig, diese in verschiedenen Datenbanksystemen 


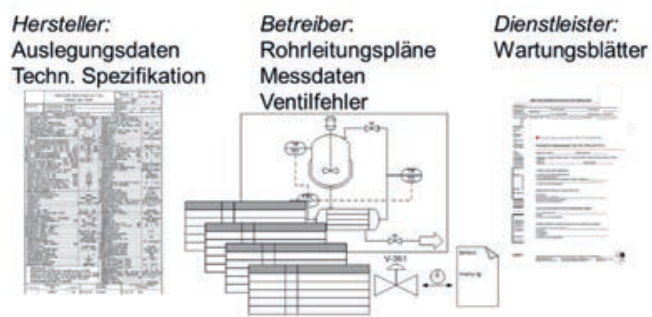

Bild 2: Relevante Datenquellen für die Ventildiagnose und beteiligte Partner bei Aufzeichnung dieser Daten.

gespeicherten, heterogenen Daten zusammenzufassen und für die Analyse zugänglich zu machen. Nur so können zum Beispiel fehlerhaft ausgelegte Ventil erkannt werden.

Innerhalb von SIDAP wird deshalb ein gemeinsames Datenmodell entwickelt, welches die zu integrierenden Datenuntermengen abbilden kann. Auf Grundlage dieses Datenmodells haben alle beteiligten Partner ein einheitliches Verständnis der Daten. Weiterhin wird durch das Datenmodell eine klare Strukturierung der Daten erzielt. Hierzu wurde auf verschiedene bereits existierende Standards und Industrieinitiativen zurückgegriffen. Dies soll die Übernahme von Daten, welche bereits in diese akzeptierten Standardformate transformiert wurden, erleichtern und somit doppelte Datenhaltung vermeiden. So versucht beispielsweise die Industrieinitiative DEXPI [3] Daten zwischen verschiedenen CAE-Werkzeugen auszutauschen. Innerhalb des SIDAP-Datenmodells dient eine modifizierte Variante von DEXPI zum Speichern der Auslegungs- und Strukturdaten der Anlage. Nur durch die Etablierung eines gemeinsamen Datenverständnisses und einer klaren Strukturierung dieser Daten können automatisierte Analysen zur Zustandsüberwachung in der Breite und flexibel eingesetzt werden.

\section{Geschlossenheit der derzeitigen IT-Systemlandschaft}

Die Struktur der Steuerungstechnik und übergeordneten Systemen ist in der industriellen Praxis sehr stark durch die klassische Automatisierungspyramide (ISA-95 [4]) geprägt. Diese Systemstruktur garantiert eine klare Trennung zwischen Prozesssteuerung und Organisation des Betriebs. Während dies für klassische Systeme, bei denen der reibungslose Betrieb der Anlage allein im Vordergrund steht, ein akzeptiertes Vorgehen, ist verlangen neuartige Anwendungen nach Veränderungen in dieser Struktur. Für den konkreten Fall der Ventildiagnose beschränkt beispielsweise die Übertragung von Messwerten über HART [5] durch die geringe Anzahl an auslesbaren Variablen die Analyse. Während moderne Stellungsregler um- 
fassende, integrierte Diagnosefunktionalitäten und eine Vielzahl auslesbarer Variablenwerte zur Verfügung stellen [6], sind per HART nur vier vorkonfigurierte Werte abrufbar. Weiterhin ist auch die Nachrüstbarkeit der Anlage mit zusätzlicher Sensorik durch die bestehende Hierarchie eingeschränkt. Da derzeit alle Signale direkt an das Prozessleitsystem übermittelt werden und diese Systeme in sich stark geschlossen sind, ist eine Erhebung zusätzlicher Signale mit enormen Aufwand verbunden.

Moderne Anwendungen, wie die Ventildiagnose, verlangen deshalb nach erweiterten, schichtenübergreifenden Wegen der Kommunikation. Hierzu muss jedoch nicht das bestehende System in seiner Gänze aufgebrochen werden. Es reicht vielmehr, einen zweiten Kommunikationskanal für nicht steuerungsrelevante Signale zu öffnen. SIDAP verfolgt hier einen ähnlichen Ansatz [7] wie er von der NAMUR in Form der Namur Open Architecture (NOA) [8] vorangetrieben wird (vergleiche Bild 3). Über den zweiten Kanal werden weitere Daten übertragen ohne das echtzeitfähige Feldbussystem und die Prozesssteuerung zu beeinflussen.

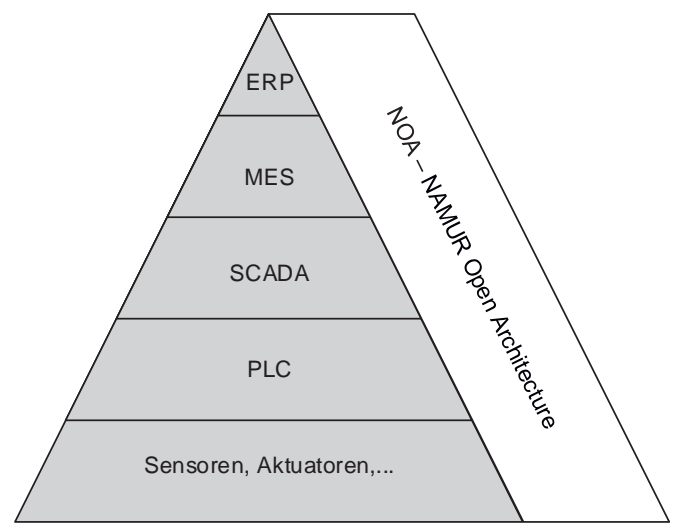

Bild 3: Schichtenübergreifende Kommunikation durch einen zweiten Kanal, hier in Form der Namur Open Architecture (angepasst nach Klettner et al. [8])

\section{Komplexität der zugrundeliegenden physikalischen Phänomene}

Die während des Drosselvorgangs auftretenden Phänomene (Kavitation, Flashing) sind komplexe fluidmechanische Vorgänge [9]. Selbst bei auftretender Kavitation durch Implosion von Dampfblasen am Ventilkegel kann nicht automatisch mit einer Schädigung des Kegels gerechnet werden. Neben dem eingesetztem Medium (Korrosivität, Abrasivität, Phasenzusammensetzung) spielt auch die Form und das Material des Ventilkegels eine entscheidende Rolle. Rein datengetriebene Analysemethoden stoßen unter diesen Umständen an Ihre 
Grenzen, da keine Berücksichtigung der spezifischen Phänomene erfolgt. Andererseits sind physikalische Modelle zur Beschreibung des Drosselvorgangs, wenn überhaupt, nur nach aufwändigen Messkampagnen erstellbar und somit nicht zur universellen Diagnose von Ventilen geeignet.

In SIDAP wurde deshalb versucht, die auftretenden Ventilschädigungen und deren zugrundeliegenden Schädigungsmechanismen zu klassifizieren. Prozessexperten und Datenanalysten erarbeiteten auf Grundlage dieses Wissens eine Schadensklassifikation, welche Schädigungen und hierfür relevante Prozessgrößen zusammenfasst [10]. Auf Basis dieser Klassifikationen können interdisziplinäre Teams anschließend hybride Modelle entwickeln, die durch richtige Auswahl der zu berücksichtigenden Parameter und der Modellierung einfacher physikalischer Zusammenhänge die geforderten Diagnosefunktionalitäten erfüllen.

\section{Sensorauswahl und Qualität der Daten}

Prozesstechnische Anlagen sind instrumentiert um einen reibungslosen Betrieb und eine Erfüllung der gesetzlichen Dokumentationspflichten zu garantieren. Unter Berücksichtigung der Komplexität der zugrundeliegenden physikalischen Phänomene wird klar, dass für eine effiziente Datenanalyse notwendige Daten oft nicht aufgezeichnet werden. So wird beispielsweise der tatsächliche Hub des Ventils oftmals nicht erfasst und lediglich der Sollwert übermittelt. Darüber hinaus wird eine Vielzahl der relevanten Messgrößen nicht im Ventil selbst erfasst, sondern durch benachbarte Sensoren aufgezeichnet. Soll die Anlage nachgerüstet werden um mehr Signale zu erfassen, müssen unter anderem oft Explosionsschutzbestimmungen berücksichtigt werden. Die bereits genannte Geschlossenheit der derzeitigen Systeme erschwert darüber hinaus die Anpassung der Instrumentierung an die gestiegenen Anforderungen durch die Datenanalyse.

Durch die Nachrüstung eines zweiten Kommunikationskanals kann die Messung der Prozessgrößen von der erweiterten Datennutzung (z.B. Datenanalyse) entkoppelt werden. Weiterhin kann die notwendige Sensorik direkt im Ventil integriert werden um bisher nicht erfasste Messsignale aufzuzeichnen. Bild 4 gibt eine innerhalb von SIDAP erfolgte Nachrüstung einer prozesstechnischen Anlage mit zusätzlicher Sensorik wieder. Prototypisch angepasste Ventile mit erweiterter Sensorik wurden neben der für die Steuerung relevanten Kommunikation über HART auch über einen zweiten Kanal in Form von WirelessHART [11] angeschlossen. Die beiden Datenkanäle werden auf dem Analysesystem wieder zusammengeführt ohne die Steuerung und das Prozessleitsystem zu beeinflussen. 


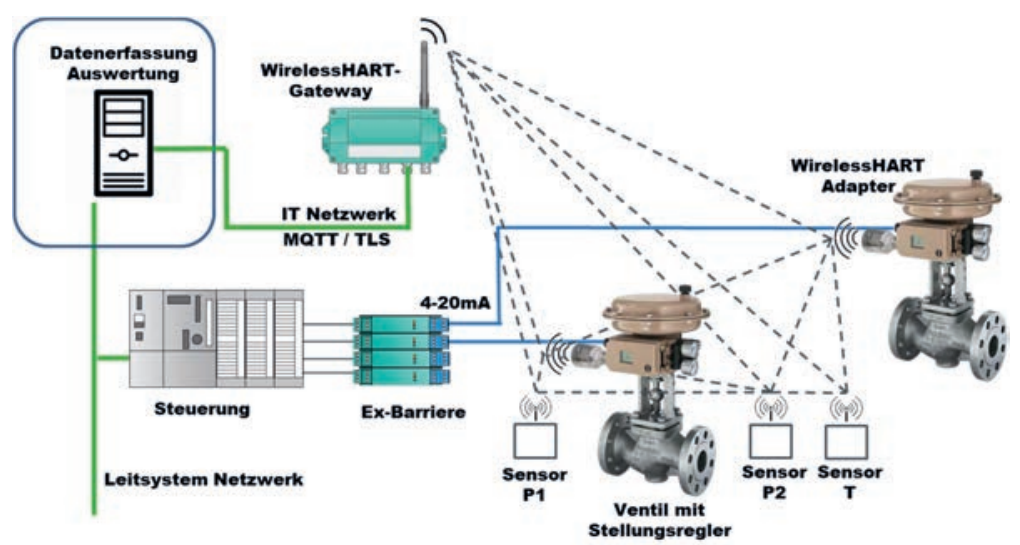

Bild 4: Nachrüstung von prozessrelevanten Regelarmaturen und Anbindung an Datenanalyse über einen zweiten Datenkanal (z.B. WirelessHART [11])

\section{Geteilte Wissensdatenbanken zur Nutzung von Synergieeffekten}

Die Zahl aufgezeichneter Ventilfehler pro Ventil ist durch die geplanten Wartungen verhältnismäßig gering. Dies erschwert die Erstellung eines Diagnosemodells, da hierfür Kenntnisse über das zu erwartende Schlechtverhalten hilfreich sind. Neben einer Berücksichtigung im Modell selbst sind die bestätigten Fehlerfälle auch zur Validierung des Modells erforderlich. Nur durch Berücksichtigung dieser Fehler kann ein zuverlässiges Modell trainiert und dessen Funktionalität nachgewiesen werden.

Durch die große Zahl an innerhalb einer Anlage oder an einem Standort eingesetzten Ventile können durch Vergleich der Betriebsbedingungen ähnliche Ventile identifiziert werden. Die Daten dieser Ventile lassen sich kombinieren, um so die benötigte Datenmenge zur Modellerstellung zu verringern. Werden die Datenbanken verschiedener Anlagenbetreiber und Armaturenhersteller in anonymisierter Form geteilt und für die Analyse kombiniert, steht eine deutlich größere Zahl an aufgezeichneten Fehlern zu Verfügung (vergleiche Bild 5). Weiterhin kann durch den Hersteller der Armatur weiteres Wissen in die Analyse eingebracht werden. Der vergrößerte Vergleichsdatensatz und die Integration dieses Expertenwissens ermöglicht die Erstellung deutlich verbesserter Modelle welche Ventilfehler mit größerer Sicherheit vorhersagen können. Andererseits profitiert der Hersteller durch Einblicke in die Betriebsweise seiner Ventile und kann so beispielsweise die Konstruktion seiner angebotenen Ventile verbessern. [1] 


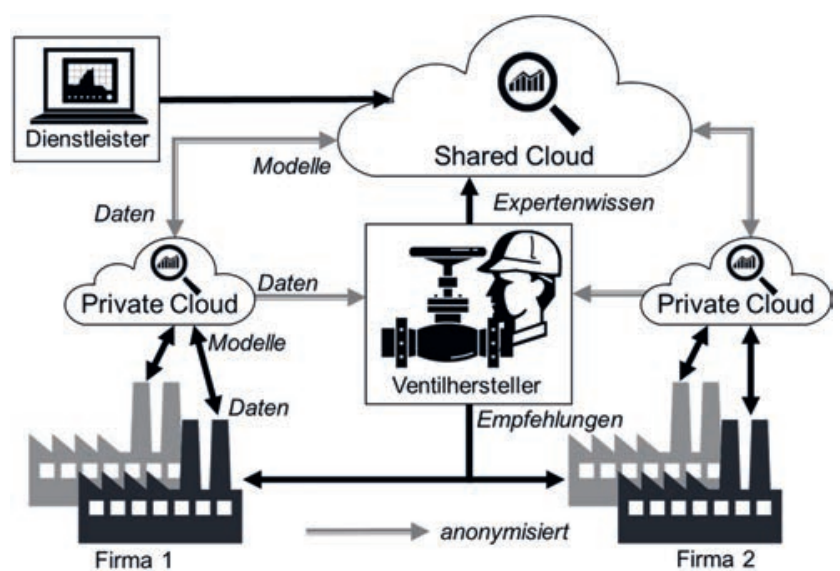

Bild 5: Geteilte Wissensdatenbanken und Cloud-Umgebung zur unternehmensübergreifenden Analyse von Daten

\section{Ventilfehlerdiagnose an einem industriellen Beispiel}

Unter Verwendung der Auslegungsgleichung aus DIN EN 60534-2-1 [12] wurde für Diagnosezwecke ein Analysemodell für die Ventildiagnose erstellt [10, 13]. Der zugrundeliegende Testdatensatz umfasst die Daten von vier ähnlichen Ventilen über 5 Jahre Laufzeit (320 Millionen Datenpunkte total). Für die Modellerstellung wurden neben den historischen Daten auch weitere Informationen aus Wartungsberichten und Auslegungsdaten berücksichtigt. Bild 6 zeigt das Ergebnis für eines der untersuchten Ventile.

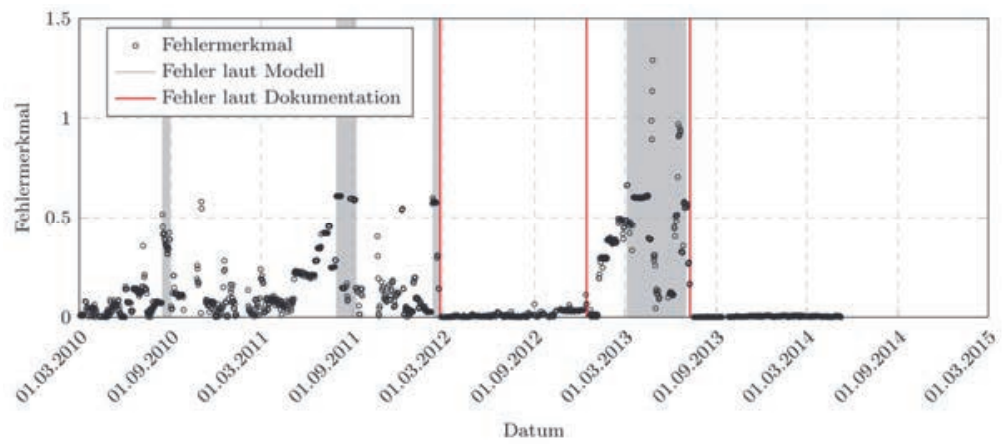

Bild 6: Beispielhaftes Ergebnis der Ventildiagnose an historischen Daten. Errechnetes Fehlermerkmal aufgetragen über der Zeit mit erkannten und dokumentierten Ventilfehlern. Nach Trunzer et al. [10]. 
Während das Modell in der Lage ist aufgezeichnete Fehler zu erkennen, werden auch Fehlalarme erzeugt. In diesen Bereichen weicht das modellierte Ventilverhalten vom gemessenen ab. Dies kann durch die nicht ausreichende Güte des Modells, aber auch durch nicht dokumentierte Fehler oder anderweitige Beeinträchtigungen des Prozesses begründet sein. Die Hinzunahme weiterer, vergleichbarer Ventile in den Datensatz kann die Erkennungsrate verbessern, da so mehr Vergleichsdaten zur Modellerstellung zur Verfügung stehen. Weiterhin müssen zusätzliche Merkmale in die Analyse mit einfließen, die den Betriebszustand des Prozesses wiedergeben, mit einbezogen werden.

\section{Zusammenfassung und Ausblick}

Die Anwendung neuer Industrie 4.0-Prinzipien und datengetriebener Analysemethoden birgt für Produktionsanlagen ein großes Potential zur Optimierung der Wirtschaftlichkeit. Der breiten industriellen Anwendung stehen jedoch noch einige Herausforderungen im Weg, welche überwunden werden müssen. Neben der Heterogenität und verteilten Speicherung der zugrundeliegenden Daten ist auch die Geschlossenheit der derzeitigen Systemlandschaft ein Problem für datengetriebene Analysen. Die Entwicklung einheitlicher Datenmodelle, welche Daten aus dem Auslegungsprozess von technischen Einrichtungen mit abbilden, und die Nutzung eines zweiten, schichtenübergreifenden Datenkanals zur Aggregation der Daten sind hier mögliche Lösungsansätze. Andererseits stehen einer rein datengetriebenen Analyse meist eine zu geringe Anzahl an Vergleichsdatensätzen, die Komplexität der zugrundeliegenden physikalischen Vorgänge und die Vollständigkeit/Qualität der Daten entgegen. Die gezielte Erfassung und Integration von Expertenwissen in den Analyseprozess kann hier zur Erstellung von hybriden Analysemodellen dienen. Weiterhin können fehlende Daten durch gezielte Nachrüstung von Anlagen mit verbesserter Sensorik und Anbindung dieser über den zweiten Datenkanal erhoben werden. Somit können Big-Data Methoden auch in bestehenden prozesstechnischen Anlagen Anwendung finden. Durch die Nutzung geteilter, unternehmensübergreifender Wissensdatenbanken kann die Menge an Vergleichsdatensätzen stark vergrößert und Synergieeffekte genutzt werden. Erst wenn die genannten Herausforderungen überwunden sind, kann das volle Potential der vorrausschauenden Wartung in der industriellen Praxis entfaltet werden.

\section{Danksagung}

Wir bedanken uns beim Bundesministerium für Wirtschaft und Energie (BMWi) für die Förderung des Projekts SIDAP (Förderkennzeichen 01MD15009F), als auch bei den Konsortialund Kooperationspartnern des Projekts. 


\section{Literaturangaben}

[1] J. Folmer et al., „Big und Smart Data: Herausforderungen in der Prozessindustrie,” atp edition, vol. 59, no. 1-2, pp. 58-69, 2017.

[2] Skalierbares Integrationskonzept zur Datenaggregation, -analyse, -aufbereitung von großen Datenmengen in der Prozessindustrie. [Online] Verfügbar: http://www.sidap.de/. Zugriff am: 06 Apr. 2018.

[3] H. Temmen, U. Argast, R. Welke, M. Theißen, und M. Wiedau, "Intelligenter R\&IAustausch: DEXPI hat einen wesentlichen Meilenstein erreicht," atp edition, vol. 58, no. 03, pp. 54-65, 2016.

[4] The Instrumentation, Systems, and Automation Society (ISA), „Enterprise-Control System Integration - Part I: Models and Terminology," ANSI/ISA 95.00.01, 2000.

[5] Samson AG, „Technische Information: HART-Kommunikation,” Frankfurt a. M., 2000.

[6] J. Kiesbauer, S. Erben, und D. Hoffmann, „Neue Asset-Management-Konzepte bei Stellventilen Diagnose von intelligenten Stellgeräten: Diagnose von intelligenten Stellgeräten," atp edition, vol. 52, no. 03, pp. 40-43, 2011.

[7] E. Trunzer, I. Kirchen, J. Folmer, G. Koltun, und B. Vogel-Heuser, „A Flexible Architecture for Data Mining from Heterogeneous Data Sources in Automated Production Systems," in 2017 IEEE International Conference on Industrial Technology (ICIT), 2017, pp. 1106-1111.

[8] C. Klettner et al., „Namur Open Architecture: Die Namur-Pyramide wird geöffnet für Industrie 4.0," atp edition, vol. 59, no. 1-2, pp. 20-37, 2017.

[9] Samson AG, "Technische Information: Kavitation in Stellventilen," Frankfurt a. M., 2003.

[10] E. Trunzer et al., „Failure mode classification for control valves for supporting datadriven fault detection," in 2017 IEEE International Conference on Industrial Engineering and Engineering Management (IEEM), Singapore, 2017, pp. 2346-2350.

[11] J. Grebner, S. Rotmensen, und R. Skowronek, „Wirless vom Feld in die Welt: Sicher drahtlos kommunizieren im Automatisierungsumfeld: WirelessHart, WLAN und Mobilfunk bieten zuverlässige Lösungen für nahezu alle Szenarien, Teil 1," atp edition, vol. 55, no. 09, pp. 22-25, 2013.

[12] Deutsches Institut für Normung e.V. (DIN), „Stellventile für die Prozessregelung - Teil 21: Durchflußkapazität - Bemessungsgleichungen für Fluide unter Betriebsbedingungen (IEC 60534-2-1:2011), “ DIN EN 60534-2-1, 2012.

[13] J. Folmer, C. Schrüfer, J. Fuchs, C. Vermum, und B. Vogel-Heuser, „Data-driven Valve Diagnosis to Increase the Overall Equipment Effectiveness in Process Industry," in 2016 IEEE 14 $4^{\text {th }}$ International Conference on Industrial Informatics (INDIN), 2016, pp. 10821087. 